\title{
Estudo anatômico do ramo infrapatelar do nervo safeno em humanos*
}

\section{Anatomical Study of the Infrapatellar Branch of the Saphenous Nerve in Humans}

\author{
Alexandre Paiva Luciano ${ }^{1,2}$ \\ Magno César Vieira ${ }^{3}$ \\ Renan Toshio Melo Honda ${ }^{2}$ Anuar Ralio Kamar ${ }^{2}$ Nelson Franco Filho ${ }^{1,2}$ \\ ${ }^{1}$ Disciplina de Ortopedia e Traumatologia, Faculdade de Medicina, \\ Universidade de Taubaté, Taubaté, SP, Brasil \\ 2 Serviço de Ortopedia e Traumatologia, Hospital Universitário de \\ Taubaté, Taubaté, SP, Brasil \\ ${ }^{3}$ Disciplina de Anatomia Descritiva e Topográfica, Faculdade de

\begin{abstract}
Endereço para correspondência Alexandre de Paiva Luciano, PhD, Serviço de Ortopedia e Traumatologia, Hospital Universitário de Taubaté, Av. Granadeiro Guimarães, 270, Centro, Taubaté, SP, 12020-130, Brasil (e-mail: paivaortopedia@gmail.com).
\end{abstract} Medicina, Universidade de Taubaté, Taubaté, SP, Brasil

Rev Bras Ortop 2020;55(5):557-563.

\section{Resumo \\ Palavras-chave \\ - joelho/inervação \\ - síndromes de compressão nervosa \\ - anatomia \\ Objetivo Realizar um estudo anatômico da localização do ramo infrapatelar do nervo safeno em relação às estruturas do joelho. \\ Métodos Estudo anatômico realizado por meio de dissecação de 18 joelhos ( 9 direitos e 9 esquerdos). Após a exposição do ramo infrapatelar e de seus ramos diretos e indiretos, seguiram-se as medidas deles. Como parâmetro do estudo, adotamos um quadrante na região medial do joelho delimitado por dois planos transversais. \\ Resultados Em 17 dos 18 joelhos estudados (94,4\%), observou-se um único ramo infrapatelar. O ramo infrapatelar emergia por entre as fibras do ventre do músculo sartório em 17 dos 18 joelhos (94,4\%). Em relação à ramificação, observamos que em $100 \%$ dos joelhos o ramo infrapatelar apresentava pelo menos uma ramificação primária, resultando num ramo superior e em outro inferior. Em 9 membros (50\% dos casos), esta ramificação ocorria fora do quadrante proposto, e, no restante, dentro do quadrante. \\ Conclusão O ramo infrapatelar do nervo safeno foi encontrado em todos os joelhos dissecados e, em $94,4 \%$ dos casos, ele era do tipo penetrante; em $100 \%$ dos casos, ele originava 2 ramos diretos primários. Seus ramos diretos e indiretos apresentaram grande variabilidade quanto ao trajeto.}

\footnotetext{
Trabalho desenvolvido na Disciplina de Anatomia Descritiva e Topográfica e Disciplina de Ortopedia e Traumatologia, Divisão de Medicina Esportiva, Departamento de Medicina da Universidade de Taubaté, Taubaté, SP, Brasil.
}

recebido

10 de Janeiro de 2019

aceito

30 de Outubro de 2019
DOI https://doi.org/

10.1055/s-0040-1701287. ISSN 0102-3616.
Copyright $(2020$ by Sociedade Brasileira License terms de Ortopedia e Traumatologia. Published by Thieme Revinter Publicações Ltda, Rio de Janeiro, Brazil 


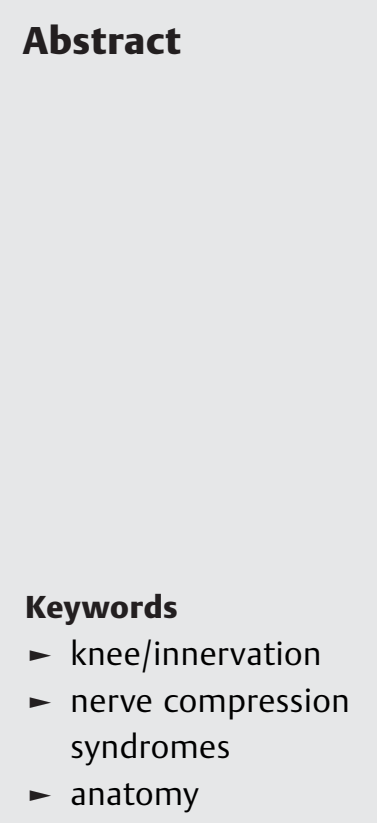

Objective To perform an anatomical study of the location of the infrapatellar branch of the saphenous nerve in relation to the structures of the knee.

Methods An anatomical study was performed by dissection of 18 humans knees ( 9 right and 9 left knees). After exposure of the infrapatellar branch and its direct and indirect branches, they were then measured. We adopted a quadrant in the medial region of the knee delimited by two transversal planes as a parameter of the study.

Results In 17 of the 18 knees (94.4\%) studied, a single infrapatellar branch was observed. The infrapatellar branch emerged as fibers of the womb of the sartorius muscle in 17 of the 18 knees (94.4\%). In relation to the branch, we observed that, in $100 \%$ of the knees, the infrapatellar branch had at least one primary branch, resulting in a superior branch and an inferior branch. In 9 limbs (50\% of the cases) this branch occurred outside the proposed quadrant, and, in the remaining limbs, it occurred within the quadrant.

Conclusion The infrapatellar saphenous nerve branch was found in all dissected knees, and, in $94.4 \%$ of the cases, it was of the penetrating type; in $100 \%$ of the cases, it originated two primary direct branches. The direct and indirect branches presented great variability regarding their path.

\section{Introdução}

A neuropatia do ramo infrapatelar do nervo safeno é uma causa frequentemente ignorada e subdiagnosticada de dor pós-operatória persistente no joelho. ${ }^{1,2}$

O ramo infrapatelar origina-se do nervo safeno, emerge distalmente ao canal adutor, perfura o músculo sartório, percorrendo um trajeto superficial, e geralmente se subdivide em dois ramos. Ambos os ramos atravessam o ligamento patelar de maneira transversal para formar o plexo infrapatelar. ${ }^{3}$ Esses pequenos ramos superficiais estão em risco de transecção, especialmente quando são realizadas incisões cirúrgicas longitudinais. A lesão no ramo infrapatelar do nervo safeno geralmente resulta em dormência no aspecto anterior do joelho, na parte lateral e proximal da perna.

Após procedimentos cirúrgicos no joelho, é comum pacientes se queixarem de dor e parestesia na região anterior do joelho, que pode irradiar-se para as regiões anterior, lateral e proximal da perna ipsilateral. Estudos também relacionam o ramo infrapatelar do nervo safeno com a inervação direta dos ligamentos mediais anteriores do joelho, sendo um dos fatores responsáveis pela propriocepção e consequente estabilidade e equilíbrio do joelho. ${ }^{3,4}$

O dano ao ramo infrapatelar do nervo safeno também foi relatado após os principais procedimentos cirúrgicos no joelho, como artroplastia total, ${ }^{5}$ artroscopia para meniscectomias, ${ }^{6}$ e pós-reconstrução do ligamento cruzado anterior. ${ }^{7}$

Assim, entendemos que o conhecimento detalhado do trajeto anatômico e das possíveis variações anatômicas do ramo infrapatelar do nervo safeno em relação às estruturas do joelho é de essencial importância para o controle das morbidades pósoperatórias, que podem variar desde parestesias até o déficit de propriocepção da articulação do joelho.

Portanto, esta pesquisa tem como objetivo realizar um estudo anatômico da localização do ramo infrapatelar do nervo safeno em relação às estruturas do joelho, com a finalidade de identificar seu trajeto, seus ramos diretos e indiretos, e o território de distribuição deles.

\section{Materiais e Métodos}

Estudo anatômico realizado por meio de dissecação de 18 joelhos (9 direitos e 9e esquerdos), sendo 11 do gênero masculino e 7 do feminino, provenientes do Laboratório de Anatomia Humana da nossa instituição. Este estudo foi previamente aprovado pelo comitê de ética (CAAE: 44657115.5.0000.5501; parecer: 1055300).

No total, 2 autores foram responsáveis pela dissecação de 18 joelhos, sendo que $77,8 \%$ (14) pertenciam a 7 cadáveres completos; $16,7 \%$ (3) eram de membros inferiores isolados, e $5,5 \%$ (1) provinham de um membro inferior amputado. Para a dissecção, foram utilizados bisturi de cabos 3 e 4, tesoura tipo Metzembaum, e pinças anatômica e dente de rato. As medidas foram realizadas com paquímetro digital Digimess (Digimess Instrumentos de Precisão Ltda. São Paulo, SP, Brasil) ( - Figura 1), e foram expressas em milímetros e confrontadas entre dois autores.

\section{Descrição de Dissecação}

A dissecação foi realizada posicionando-se o cadáver em decúbito dorsal horizontal com o joelho em extensão. Foram realizadas três incisões na pele. Duas incisões horizontais: a primeira, no terço inferior e distal da coxa, e a segunda, entre os terços superior e médio da perna. Fez-se uma incisão vertical unindo os pontos médios das incisões horizontais, coincidentes, na posição superoinferior, com o ponto médio da base da patela, o ápice da patela, e a tuberosidade da tíbia. Em seguida, foi realizado o rebatimento cutâneo de lateral para medial, expondo a tela subcutânea, na qual foi dissecado o ramo infrapatelar do nervo safeno e seus ramos diretos e indiretos (-Figura 2). 


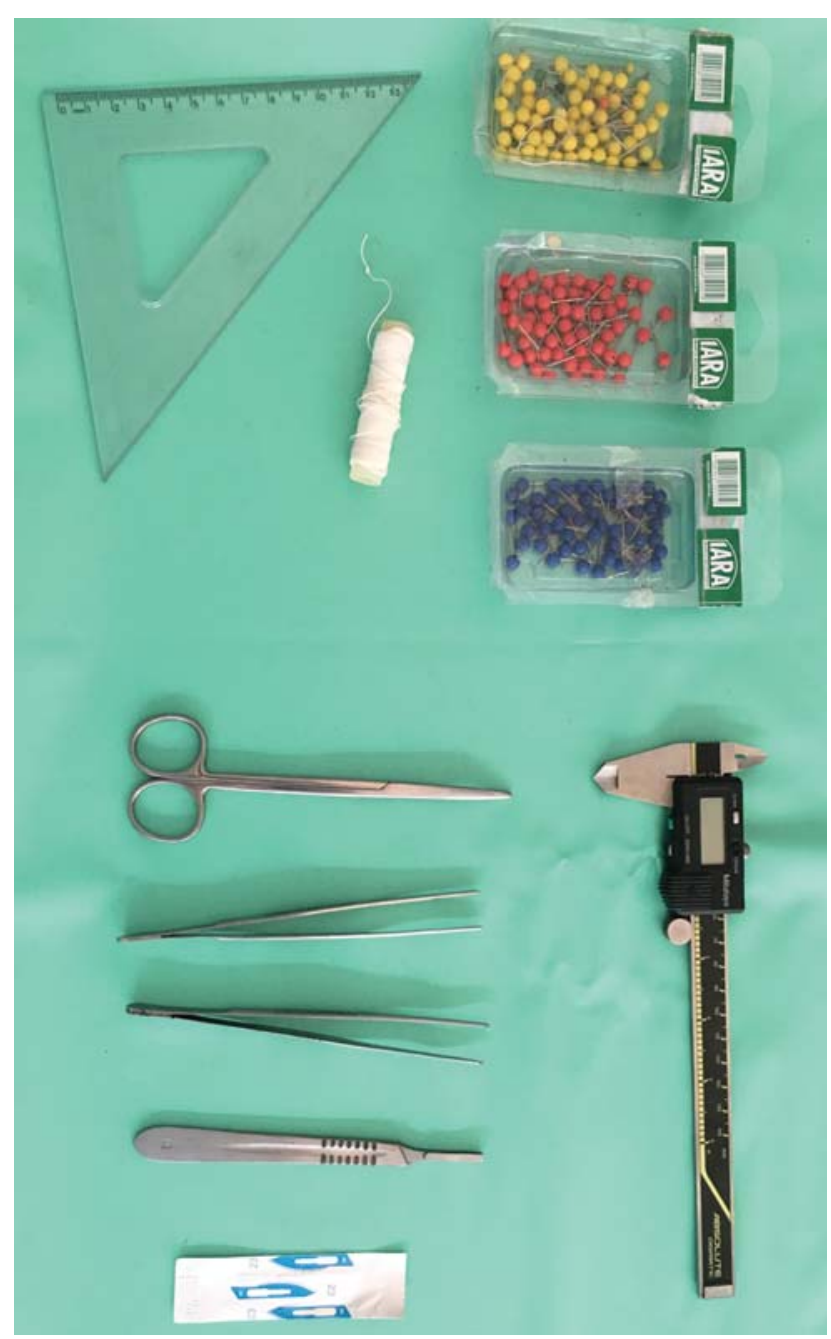

Fig. 1 Material utilizado para dissecação: bisturi de cabos 3 e 4, tesoura tipo Metzembaum, pinças anatômica e dente de rato, e paquímetro digital Digimess.

Após a exposição do ramo infrapatelar e de seus ramos diretos e indiretos, seguiram-se as medidas deles. Para tal, adotamos um quadrante (área do estudo) na região medial do joelho delimitado por dois planos transversais: um superior, que passava pela linha articular (LA) do joelho, e outro inferior, que cruzava o ponto mais alto da tuberosidade da tíbia, chamado linha do tubérculo tibial (LTT), e uma linha longitudinal (LL), que cruzava perpendicularmente os planos transversais, desde o ponto médio do platô tibial até o ponto mais alto da tuberosidade da tíbia (- Figura $\mathbf{3}$ ).

Os pontos de referência do platô tibial e da tuberosidade da tíbia, respectivamente, superior e inferiormente, foram demarcados com alfinetes coloridos. Com um esquadro, marcou-se o ponto de cruzamento das linhas, definindo o quadrante para o estudo (-Figura 4).

\section{Nomenclatura Utilizada para as Medidas (-Figura 4)}

Medida 1: distância entre o ponto de emergência do ramo infrapatelar na região medial do joelho e a LA;

Medida 2: distância entre o ponto de ramificação primária do ramo infrapatelar e a LA e a LL;

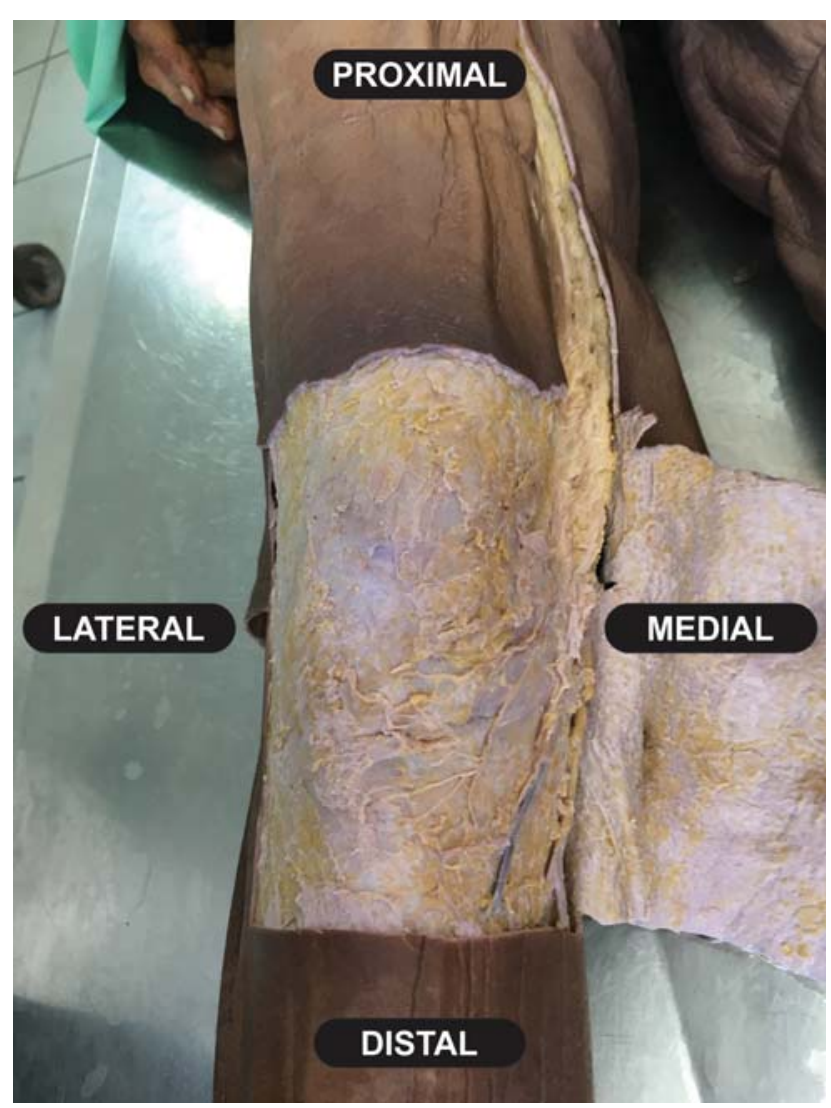

Fig. 2 Incisões da pele com rebatimento cutâneo de lateral para medial, nas quais foram dissecados o ramo infrapatelar do nervo safeno e seus ramos diretos e indiretos.

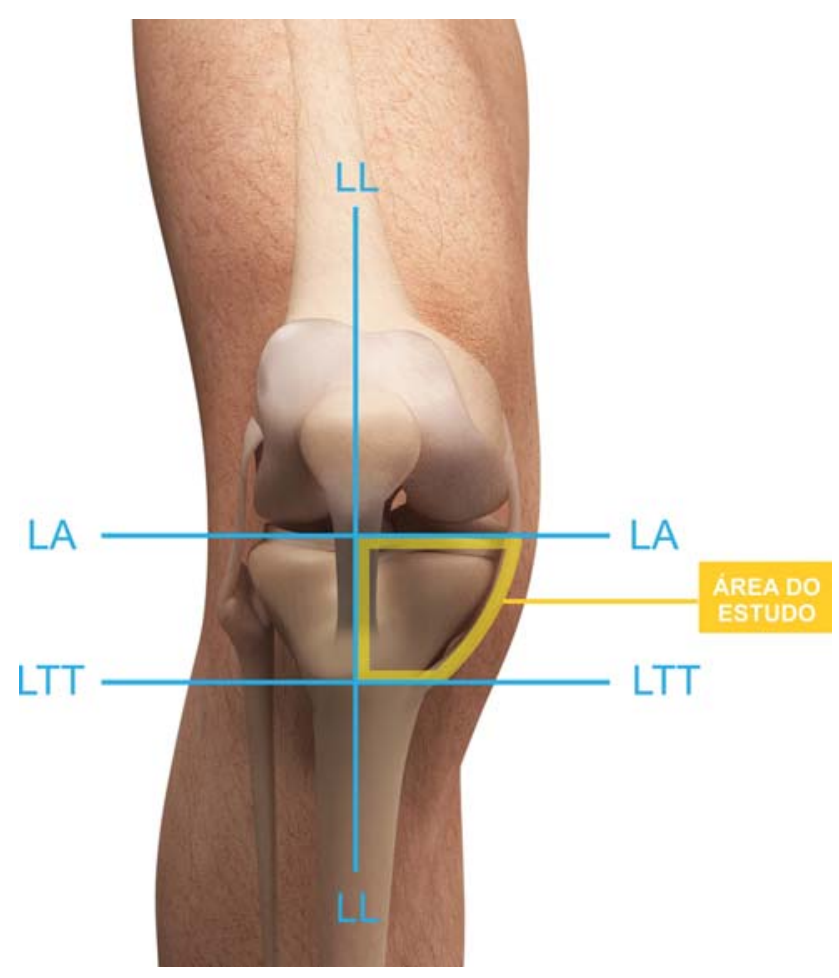

Fig. 3 Linhas de referência: linha articular (LA), linha do tubérculo tibial (LTT), e linha longitudinal (LL), utilizadas para estabelecer o quadrante (área) do estudo. 


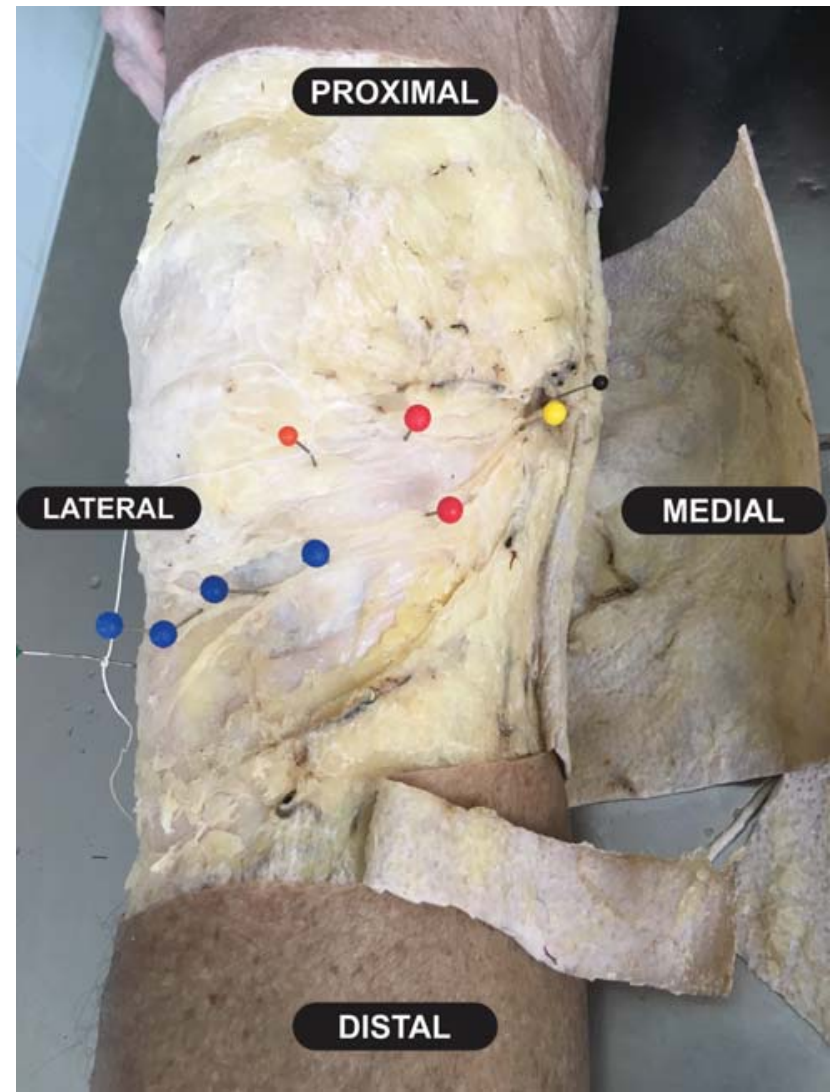

Fig. 4 Pontos de referência do platô tibial e tuberosidade da tíbia, respectivamente, superior e inferiormente, demarcados com alfinetes coloridos.

Medida 3: distância entre as ramificações secundárias, chamadas de "a," "b" e "c" de acordo com a sua aparição, e a LA e a LL;

Medida 4: distância entre as ramificações terciárias, chamadas de "a," "b" e "c" de acordo com a sua aparição, e a LA e a LL.

Os dados obtidos de cada joelho foram organizados em tabelas, com as suas devidas identificações (gênero, lado e se do mesmo indivíduo, membro isolado ou amputado) e medidas de acordo com os critérios adotados e descritos (-Figura 5).

\section{Resultados}

Em 17 dos 18 joelhos (94,4\%) estudados, observou-se um único ramo infrapatelar. Uma subdivisão proximal do ramo infrapatelar foi observada em um único joelho.

0 ramo infrapatelar emergia por entre as fibras proximais do ventre do músculo sartório em 17 dos 18 joelhos (94,4\%), sendo que, em 1 joelho, não foi possível a sua identificação completa no local de origem descrito.

A - Tabela 1 mostra a distribuição da distância entre o ponto de emergência em relação à LA; estas medidas demonstraram grande variação. Em 9 joelhos, num total de 10 ramos infrapatelares, a medida 1 ocorreu fora do quadrante proposto no estudo, sendo a maior distância de $200,14 \mathrm{~mm}$, e a menor, de $2,81 \mathrm{~mm}$, com uma média de

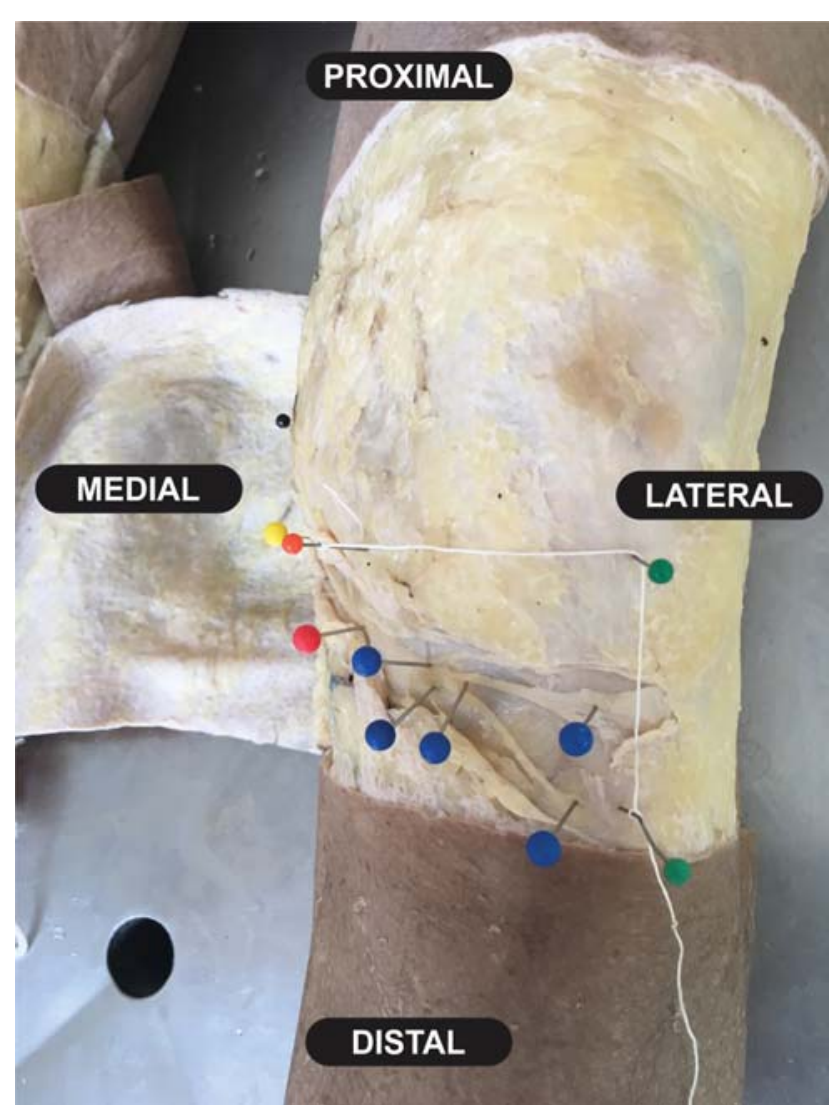

Fig. 5 Distribuição da distância entre o ponto de emergência em relação à $\mathrm{LA}$ e à $\mathrm{LL}$, demonstrando grande variação.

$83,14 \mathrm{~mm}$. Em 8 joelhos, o ramo infrapatelar tinha origem dentro do quadrante proposto, sendo que a distância distal em relação à LA foi de $44,85 \mathrm{~mm}$, e a proximal, de $5,64 \mathrm{~mm}$, com média de $37,80 \mathrm{~mm}$. Entre os sete indivíduos nos quais observamos ambos os joelhos, quatro apresentavam o ponto de emergência do ramo infrapatelar fora e dentro do quadrante proposto; em dois indivíduos, a emergência era fora do quadrante em ambos os joelhos; e, em apenas um indivíduo, a emergência do ramo infrapatelar, em ambos os joelhos, era dentro do quadrante.

A - Tabela 2 mostra as medidas das distâncias entre os pontos de ramificação primária, secundária e terciária do interior do quadrante estabelecido segundo a LA e a LL.

Em relação à ramificação, observamos que em 100\% dos joelhos o ramo infrapatelar apresentava pelo menos uma ramificação primária, resultando num ramo superior e em outro inferior. Em 9 membros (50\% dos casos), esta ramificação ocorria fora do quadrante proposto, e, no restante, dentro do quadrante, sendo que, no joelho com dois ramos infrapatelares, as ramificações primárias de ambos os ramos ocorriam no interior do quadrante.

A maior distância entre a ramificação primária dos 10 ramos infrapatelares que se dividem no interior do quadrante à LA foi de $64,23 \mathrm{~mm}$, e a menor, de 2,9 mm, com uma média de $26,51 \mathrm{~mm}$. Já em relação à $\mathrm{LL}$, a maior distância foi de $86,07 \mathrm{~mm}$, e a menor, de $59,61 \mathrm{~mm}$, com uma média de 73,53 $\mathrm{mm}$ (-Tabela 2). 
Tabela 1 Distância em milímetros do ponto de emergência do ramo do nervo safeno em relação aos quadrantes determinados

\begin{tabular}{|l|l|l|}
\hline Indivíduos & \multicolumn{2}{|l|}{$\begin{array}{l}\text { Distância (mm) - ponto } \\
\text { de emergência em } \\
\text { relação à linha articular }\end{array}$} \\
\hline & $\begin{array}{l}\text { Fora do } \\
\text { quadrante }\end{array}$ & $\begin{array}{l}\text { Dentro do } \\
\text { quadrante }\end{array}$ \\
\hline Indivíduo 1 - Joelho D Masc & 61,86 & - \\
\hline Indivíduo 1 - Joelho E Masc & 112,15 & - \\
\hline Indivíduo 2 - Joelho D Masc & 182,03 & - \\
\hline Indivíduo 2 - Joelho E Masc & - & 11,42 \\
\hline Indivíduo 3 - Joelho D Masc & - & 5,84 \\
\hline Indivíduo 3 - Joelho E Masc & - & 44,85 \\
\hline Indivíduo 4 - Joelho D Masc & - & 26,78 \\
\hline Indivíduo 4 - Joelho E Masc & 30,5 & - \\
\hline Indivíduo 5 - Joelho D Masc & - & 5,64 \\
\hline Indivíduo 6 - Joelho E Masc & - & 29,2 \\
\hline Indivíduo 7 - Joelho E Masc N & $20,50 N /$ & - \\
\hline Indivíduo 8 - Joelho D Fem & 134,88 & - \\
\hline Indivíduo 8 - Joelho E Fem & 200,14 & - \\
\hline Indivíduo 9 - Joelho D Fem & - & 13,74 \\
\hline Indivíduo 9 - Joelho E Fem & 70,66 & - \\
\hline Indivíduo 10 - Joelho D Fem & - & 13,74 \\
\hline Indivíduo 10 - Joelho E Fem & 2,81 & - \\
\hline Indivíduo 11 - Joelho D Fem & - & - \\
\hline & & \\
\hline
\end{tabular}

Abreviaturas: D, direito; E, esquerdo; Fem, feminino; Masc, masculino; $\mathrm{N}, \mathrm{N}^{*}$, subdivisões proximais do ramo infrapatelar observadas em um único joelho.

Observamos na - Tabela 2 que, em 11 joelhos $(61,1 \%)$, as ramificações secundárias ocorriam dentro do quadrante, sendo que, em 8 casos (72,7\%) esta ramificação era proveniente de uma ramificação primária no interior do quadrante, e, em 3 casos (27,3\%), ela ocorria fora do quadrante estudado. Observou-se também que, dos 11 joelhos, 6 (54,5\%) apresentavam duas ramificações secundárias, que foram denominadas “a” e "b", resultando em 4 ramos; 3 (27,3\%) apresentavam 1 única ramificação, originando 2 ramos; em 1 joelho $(9,1 \%)$, notaram-se 3 ramificações secundárias, denominadas “a”, "b” e "c", que resultaram em 6 ramos secundários; e em outro joelho (9,1\%), observaram-se 5 ramificações, denominadas “a”, "b”, “c”, "d" e "e", que resultaram 10 ramos secundários.

Em relação à LA, a ramificação secundária mais distal estava a $65,65 \mathrm{~mm}$, e a mais proximal, a $2,34 \mathrm{~mm}$, com um valor médio de $31,87 \mathrm{~mm}$. Em relação à $\mathrm{LL}$, a distância mais medial estava a $86,54 \mathrm{~mm}$, e a mais lateral, a $17,57 \mathrm{~mm}$, com uma média de $48,40 \mathrm{~mm}$.

Na - Tabela 2, nota-se também que, em 7 (38,9\%) dos 18 joelhos estudados, foram observadas ramificações terciárias do ramo infrapatelar, sendo que, em 5 membros inferiores $(71,4 \%)$, estes ramos erram procedentes de ramificações secundárias presentes no quadrante, e, em 2 casos $(28,6 \%)$, de ramos secundários situados fora do quadrante. Em relação ao número de ramificações terciárias, observou-se que, em 3 $(42,8 \%)$ dos 7 joelhos, havia 2 ramificações; em 2 (28,6\%), 3 ramificações; em 1, (14,3\%), 4 ramificações; e, em 1 (14,3\%) outro, apenas uma ramificação.

O ponto de origem dos ramos terciários, mais distante da LA, encontrava-se a 73,62 mm, e o mais próximo, a 0,92 mm, com um valor médio de $46,33 \mathrm{~mm}$. Em relação à LL, o ponto mais medial estava a $51,57 \mathrm{~mm}$, e o mais lateral, a $12,30 \mathrm{~mm}$, com uma média de $32,98 \mathrm{~mm}$.

Pudemos observar que 3 (16,7\%) dos 18 joelhos estudados tinham as ramificações primárias, secundárias e terciárias no interior do quadrante proposto.

Observamos ainda que, dos 9 casos em que a ramificação primária ocorria fora do quadrante, em 7 (77,8\%) ela ocorria acima da LA, e, em 2 (22,2\%) casos, ela ocorria abaixo da LTT.

Em relação aos 18 joelhos, observou-se que, em 5 (27,8\%) casos, as ramificações secundárias cruzavam a LL, e, em 2 $(11,1 \%)$ casos, eram as ramificações terciárias que a ultrapassavam, inervando a região lateral do joelho; as ramificações terciárias, em $2(11,1 \%)$ casos, cruzavam a LTT para se distribuir abaixo da tuberosidade da tíbia, e, em 9 (50,0\%) dos casos estudados, observou-se que as ramificações secundárias e terciárias cruzavam tanto a LL quanto a LTT.

Em relação ao quadrante proposto, o ramo infrapatelar não se restringia aos limites propostos em nenhum joelho avaliado. Porém, além dos seus limites, o ramo infrapatelar alcançava a região abaixo da LTT em 11 (61,1\%) joelhos, chegava acima da LA em 9 (50\%) casos, e se estendia desde a parte de cima da LA até abaixo da LTT em 6 (33,3\%) joelhos.

\section{Discussão}

A importância do estudo do ramo infrapatelar do nervo safeno está bem estabelecida na literatura, e sua lesão pode levar desde a parestesias leves, anestesia, formação de neuroma até distrofia simpática reflexa. ${ }^{8,9}$

Em nosso estudo, o ramo infrapatelar foi observado em todos os joelhos dissecados, sendo que, em um único joelho, foi constatada a presença de dois ramos infrapatelares, o que está de acordo com a literatura, que também é clara quanto à presença do ramo infrapatelar do nervo safeno. ${ }^{10-12}$ Para alguns autores, ${ }^{3,11}$ o ramo infrapatelar originou 2 troncos, um superior e outro inferior, assim como em nosso estudo, no qual constatamos a presença de 2 ramos oriundos do ramo infrapatelar em $100 \%$ dos casos.

Os resultados desta pesquisa também mostram que, de acordo com a literatura, a variação anatômica do ramo infrapatelar do nervo safeno é alta. ${ }^{3,13,14}$ Pesquisas ${ }^{15,16}$ identificam, em média, de três a quatro tipos diferentes de ramo infrapatelar em relação ao músculo sartório: anterior, posterior, penetrante e pes anserinus. Em nossos achados, observamos que, em 17 (94,4\%) casos, o ramo infrapatelar era do tipo penetrante, ou seja, emergia por entre as fibras musculares do ventre do músculo sartório.

Kalthur et al. ${ }^{16}$ em seu estudo de 32 joelhos, mostraram que a distribuição dos ramos foi observada: entre o ápice da patela e a tuberosidade da tíbia em 17 casos; no nível da 
562 Estudo anatômico do ramo infrapatelar do nervo safeno em humanos Luciano et al.

Tabela 2 Distância em milímetros das principais ramificações em relação à linha articular (LA) e à linha longitudinal (LL)

\begin{tabular}{|c|c|c|c|c|c|c|}
\hline \multirow[t]{2}{*}{ Indivíduos } & \multicolumn{2}{|c|}{$\begin{array}{l}\text { Distância }(\mathrm{mm}) \\
\text { ramificação } \\
\text { primária - LA/LL }\end{array}$} & \multicolumn{2}{|c|}{$\begin{array}{l}\text { Distância }(\mathrm{mm}) \\
\text { ramificação } \\
\text { secundária - LA/LL }\end{array}$} & \multicolumn{2}{|c|}{$\begin{array}{l}\text { Distância }(\mathrm{mm}) \\
\text { ramificação } \\
\text { terciária - LA/LL }\end{array}$} \\
\hline & LA & $\overline{L L}$ & LA & LL & LA & $\overline{L L}$ \\
\hline \multirow[t]{2}{*}{ Indivíduo 1 - Joelho D Masc } & A: 15,90 & A: 71,50 & A: 11,94 & A: 35,36 & & \\
\hline & & & B: 31,58 & B: 38,65 & & \\
\hline \multirow[t]{2}{*}{ Indivíduo 1 - Joelho E Masc } & A: 2,90 & A: 60,50 & $A: 27,85$ & A: 58,87 & & \\
\hline & & & $\mathrm{B}: 28,12$ & $\mathrm{~B}: 42,88$ & & \\
\hline \multirow[t]{3}{*}{ Indivíduo 2 - Joelho D Masc } & & & A: 2,34 & A: 60,55 & A: 24,54 & A: 39,57 \\
\hline & & & & & B: 49,90 & B: 38,97 \\
\hline & & & & & C: 53,08 & C: 37,07 \\
\hline \multirow[t]{3}{*}{ Indivíduo 2 - Joelho E Masc } & A: 17,24 & A: 85,49 & A: 45,81 & A: 58,89 & A: 68,98 & A: 33,60 \\
\hline & & & B: 45,61 & B: 54,46 & & \\
\hline & & & $C: 50,95$ & C: 49,31 & & \\
\hline Indivíduo 3 - Joelho D Masc & A: 24,17 & A: 81,15 & A: 42,24 & A: 74,94 & & \\
\hline \multicolumn{7}{|l|}{ Indivíduo 3 - Joelho E Masc } \\
\hline \multicolumn{7}{|l|}{ Indivíduo 4 - Joelho D Masc } \\
\hline \multirow[t]{2}{*}{ Indivíduo 4 - Joelho E Masc } & & & & & A: 23,81 & A: 45,05 \\
\hline & & & & & B: 30,28 & B: 45,21 \\
\hline \multirow[t]{2}{*}{ Indivíduo 5 - Joelho D Masc } & A: 20,9 & A: 86,1 & A: 22,89 & A: 38,80 & & \\
\hline & & & B: 22,60 & B: 27,91 & & \\
\hline Indivíduo 6 - Joelho E Masc & A: 64,23 & A: 76,06 & & & & \\
\hline \multirow[t]{2}{*}{ Indivíduo 7 - Joelho E Masc N } & $\begin{array}{l}\text { A: } 23,92 \text { N/A: } \\
26,46 N^{*}\end{array}$ & $\begin{array}{l}\text { A: } 63,84 \mathrm{~N} / \mathrm{A}: \\
76,06 \mathrm{~N}^{*}\end{array}$ & A: 43,18 & A: 28,91 & A: 47,21 & A: 13,00 \\
\hline & & & & & B: 52,01 & B: 12,30 \\
\hline \multirow[t]{3}{*}{ Indivíduo 8 - Joelho D Fem } & A: 28,82 & A: 59,61 & $A: 31,52$ & A: 58,57 & A: 54,93 & A: 39,65 \\
\hline & & & B: 39,03 & B: 63,14 & B: 54,97 & B: 36,65 \\
\hline & & & & & C: 60,23 & C: 31,49 \\
\hline \multirow[t]{3}{*}{ Indivíduo 8 - Joelho E Fem } & & & A: 8,88 & A: 86,54 & A: 57,75 & A: 51,57 \\
\hline & & & B: 41,12 & B: 63,69 & B: 73,62 & B: 37,39 \\
\hline & & & & & C: 68,08 & C: 22,24 \\
\hline \multicolumn{7}{|l|}{ Indivíduo 9 - Joelho D Fem } \\
\hline \multirow[t]{3}{*}{ Indivíduo 9 - Joelho E Fem } & & & A: 16,20 & A: 57,68 & & \\
\hline & & & B: 21,94 & $\mathrm{~B}: 38,17$ & & \\
\hline & & & $C: 23,08$ & C: 19,69 & & \\
\hline \multicolumn{7}{|l|}{ Indivíduo 10 - Joelho D Fem } \\
\hline \multirow[t]{2}{*}{ Indivíduo 10 - Joelho E Fem } & A: 40,64 & A: 75,06 & $A: 49,56$ & A: 75,23 & & \\
\hline & & & B: 65,65 & B: 42,51 & & \\
\hline \multirow[t]{2}{*}{ Indivíduo 11 - Joelho D Fem } & & & & & A: 0,92 & A: 22,22 \\
\hline & & & & & B: 12,82 & B: 25,71 \\
\hline
\end{tabular}

Abreviaturas: A, B, C, número de ramificações secundárias encontradas durante a dissecação; D, direito; E, esquerdo; Fem, feminino; Masc, masculino; $\mathrm{N}, \mathrm{N}^{*}$, subdivisões proximais do ramo infrapatelar observadas em um único joelho.

tuberosidade da tíbia em 5 casos; e no nível da patela em 1 caso; os casos remanescentes tinham uma distribuição variável. Em nosso estudo, encontramos um total de 114 ramos, diretos e/ou indiretos, presentes dentro do quadrante proposto. Dos 18 joelhos estudados, não foi observado nenhum ramo no nível da patela, nem ramificação estritamente acima da LA. Em dois casos, a distribuição dos ramos ocorria abaixo da LT. Nos demais casos, apresentava-se abaixo da LA, com distribuição variável, mas com um trajeto distal-lateral. 
Nesta pesquisa, a maior distância entre a ramificação primária dos 10 ramos infrapatelares que se dividem no interior do quadrante proposto à LA foi de $64,23 \mathrm{~mm}$, e a menor, de 2,9 mm, com um média de $26,51 \mathrm{~mm}$. Já em relação à $L L$, a maior distância foi de $86,07 \mathrm{~mm}$, e a menor, de $59,61 \mathrm{~mm}$, com uma média de 73,53 mm. Não foi observado um padrão de inervação restrito ao sexo ou aos membros do mesmo indivíduo, o que reforça a dificuldade de encontrar uma incisão ideal em procedimentos cirúrgicos na região do joelho. Apesar da grande variação, os troncos principais foram todos localizados na região medial do joelho no nível do platô tibial, próximo à LA medial, com ramificação em sentido horizontal em direção ao tendão patelar, próximo à LL. Portanto, podemos estabelecer uma zona anteromedial de maior risco para incisões cirúrgicas no joelho. A literatura ressalta que incisões da pele na região anteromedial fornecem uma excelente exposição cirúrgica, e minimizam o comprimento da incisão. No entanto, também estão associadas, em 55\% a 100\% dos casos, com persistência de uma área de hipoestesia na face anterior do joelho. ${ }^{17-21}$

Outros estudos 3,6,10,11 também delimitaram zonas de maior e/ou menor risco para incisões cirúrgicas em relação ao ramo infrapatelar do nervo safeno em volta do joelho. Kerver et al. ${ }^{3}$ estabeleceram três zonas distintas, consideradas de baixa morbidade, com menor densidade de ramos infrapatelares do nervo safeno, teoricamente seguras e com menor morbidade para incisões cirúrgicas. A primeira zona estaria localizada no lado medial do joelho, no nível da tuberosidade anterior da tíbia, onde uma incisão oblíqua é menos propensa a danificar o ramo infrapatelar, e as outras duas zonas estão localizadas medialmente ao ápice da patela, e, em ambas as incisões mais horizontais, são menos propensas a danificar o ramo infrapatelar.

\section{Conclusão}

Assim, podemos concluir que ramo infrapatelar do nervo safeno foi encontrado em todos os joelhos dissecados e, na maioria dos casos, era do tipo penetrante, originando dois ramos diretos primários: um superior e outro inferior em todos os joelhos dissecados. O ramo infrapatelar do nervo safeno apresentou grande variabilidade quanto ao seu trajeto e suas ramificações diretas e indiretas, inervando a região entre a LA do joelho e tuberosidade anterior da tíbia por meio de seus ramos, havendo, assim, poucos subsídios para a padronização do seu comportamento. Isso faz com que o ramo infrapatelar do nervo safeno corra o risco de sofrer danos em qualquer procedimento cirúrgico no aspecto anteromedial do joelho.

\section{Conflito de Interesses}

Os autores declaram não haver conflito de interesses.

\section{Referências}

1 Tennent TD, Birch NC, Holmes MJ, Birch R, Goddard NJ. Knee pain and the infrapatellar branch of the saphenous nerve. J R Soc Med 1998;91(11):573-575
2 Lippitt AB. Neuropathy of the saphenous nerve as a cause of knee pain. Bull Hosp Jt Dis 1993;52(02):31-33

3 Kerver AL, Leliveld MS, den Hartog D, Verhofstad MH, Kleinrensink GJ. The surgical anatomy of the infrapatellar branch of the saphenous nerve in relation to incisions for anteromedial knee surgery. J Bone Joint Surg Am 2013;95(23):2119-2125

4 Katz MM, Hungerford DS. Reflex sympathetic dystrophy affecting the knee. J Bone Joint Surg Br 1987;69(05):797-803

5 Sundaram RO, Ramakrishnan M, Harvey RA, Parkinson RW. Comparison of scars and resulting hypoaesthesia between the medial parapatellar and midline skin incisions in total knee arthroplasty. Knee 2007;14(05):375-378

6 Mochida H, Kikuchi S. Injury to infrapatellar branch of saphenous nerve in arthroscopic knee surgery. Clin Orthop Relat Res 1995;(320): 88-94

7 Papastergiou SG, Voulgaropoulos H, Mikalef P, Ziogas E, Pappis G, Giannakopoulos I. Injuries to the infrapatellar branch(es) of the saphenous nerve in anterior cruciate ligament reconstruction with four-strand hamstring tendon autograft: vertical versus horizontal incision for harvest. Knee Surg Sports Traumatol Arthrosc 2006;14(08):789-793

8 Poehling GG, Pollock FE Jr, Koman LA. Reflex sympathetic dystrophy of the knee after sensory nerve injury. Arthroscopy 1988;4 (01):31-35

9 Katsoulis E, Court-Brown C, Giannoudis PV. Incidence and aetiology of anterior knee pain after intramedullary nailing of the femur and tibia. J Bone Joint Surg Br 2006;88(05):576-580

10 Ebraheim NA, Mekhail AO. The infrapatellar branch of the saphenous nerve: an anatomic study. J Orthop Trauma 1997;11(03):195-199

11 Tifford CD, Spero L, Luke T, Plancher KD. The relationship of the infrapatellar branches of the saphenous nerve to arthroscopy portals and incisions for anterior cruciate ligament surgery. An anatomic study. Am J Sports Med 2000;28(04):562-567

12 Gali JC, Resina AF, Pedro G, et al. Importância da localização anatômica do ramo infrapatelar do nervo safeno na reconstrução do ligamento cruzado anterior com tendões flexores. Rev Bras Ortop 2014;49(06):625-629

13 Kartus J, Ejerhed L, Eriksson BI, Karlsson J. The localization of the infrapatellar nerves in the anterior knee region with special emphasis on central third patellar tendon harvest: a dissection study on cadaver and amputated specimens. Arthroscopy 1999; 15(06):577-586

14 Horner G, Dellon AL. Innervation of the human knee joint and implications for surgery. Clin Orthop Relat Res 1994;(301):221-226

15 Ackmann T, Von Düring M, Teske W, Ackermann O, Muller P, Von Schulze Pellengahr C. Anatomy of the infrapatellar branch in relation to skin incisions and as the basis to treat neuropathic pain by cryodenervation. Pain Physician 2014;17(03): E339-E348

16 Kalthur SG, Sumalatha S, Nair N, Pandey AK, Sequeria S, Shobha L. Anatomic study of infrapatellar branch of saphenous nerve in male cadavers. Ir J Med Sci 2015;184(01):201-206

17 Laffosse JM, Potapov A, Malo M, Lavigne M, Vendittoli PA. Hypesthesia after anterolateral versus midline skin incision in TKA: a randomized study. Clin Orthop Relat Res 2011;469(11): 3154-3163

18 Borley NR, Edwards D, Villar RN. Lateral skin flap numbness after total knee arthroplasty. J Arthroplasty 1995;10(01):13-14

19 Hopton BP, Tommichan MC, Howell FR. Reducing lateral skin flap numbness after total knee arthroplasty. Knee 2004;11(04): 289-291

20 Spicer DD, Blagg SE, Unwin AJ, Allum RL. Anterior knee symptoms after four-strand hamstring tendon anterior cruciate ligament reconstruction. Knee Surg Sports Traumatol Arthrosc 2000;8(05):286-289

21 Quayle JB, Robinson MP. An operation for chronic prepatellar bursitis. J Bone Joint Surg Br 1976;58-B(04):504-506 